\title{
DISCOVERY OF A DYNAMICAL COLD POINT IN THE HEART OF THE SAGITTARIUS dSph GALAXY WITH OBSERVATIONS FROM THE APOGEE PROJECT
}

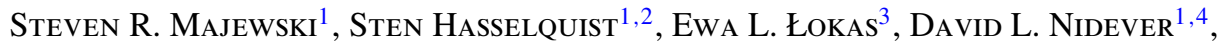 \\ Peter M. Frinchaboy ${ }^{5}$, Ana E. García Pérez ${ }^{1}$, Kathryn V. Johnston ${ }^{6}$, Szabolcs Mészáros ${ }^{7,8}$, Matthew Shetrone ${ }^{9}$, \\ Carlos Allende Prieto ${ }^{7,8}$, Rachael L. Beaton ${ }^{1}$, Timothy C. Beers ${ }^{10,11}$, Dmitry Bizyaev ${ }^{12}$, Katia Cunha ${ }^{10,13,14}$, \\ Guillermo Damke ${ }^{1}$, Garrett Ebelke ${ }^{12}$, Daniel J. Eisenstein ${ }^{15}$, Fred Hearty ${ }^{1}$, Jon HoltzMan ${ }^{2}$, Jennifer A. Johnson ${ }^{16}$, \\ David R. LaW ${ }^{17}$, Viktor Malanushenko ${ }^{12}$, Elena Malanushenko ${ }^{12}$, Robert W. O'Connell ${ }^{1}$, Daniel Oravetz $^{12}$, \\ Kaike Pan $^{12}$, Ricardo P. Schiavon ${ }^{18,19}$, Donald P. Schneider ${ }^{20,21}$, Audrey Simmons ${ }^{12}$, Michael F. Skrutskie ${ }^{1}$, \\ Verne V. Smith ${ }^{10}$, John C. Wilson $^{1}$, And Gail ZasowsKi ${ }^{1,22}$ \\ ${ }^{1}$ Department of Astronomy, University of Virginia, Charlottesville, VA 22904-4325, USA; srm4n@ virginia.edu, sh6cy@ virginia.edu, aeg4x@ virginia.edu, \\ rlb9n@virginia.edu, gjd3r@virginia.edu, frh3z@virginia.edu, rwo@virginia.edu, jcw6z@virginia.edu \\ ${ }^{2}$ New Mexico State University, Las Cruces, NM 88003, USA; holtz@ nmsu.edu \\ ${ }^{3}$ Nicolaus Copernicus Astronomical Center, Bartycka 18, 00-716 Warsaw, Poland; lokas@camk.edu.pl \\ ${ }^{4}$ Department of Astronomy, University of Michigan, Ann Arbor, MI 48109, USA; dnidever@umich.edu \\ ${ }^{5}$ Texas Christian University, Fort Worth, TX 76129, USA; p.frinchaboy@ tcu.edu \\ ${ }^{6}$ Department of Astronomy, Columbia University, Mail Code 5246, New York, NY 10027, USA; kvj@astro.columbia.edu \\ ${ }^{7}$ Instituto de Astrofísica de Canarias, E-38205 La Laguna, Tenerife, Spain; meszi@iac.es, callende@iac.es \\ ${ }^{8}$ Departamento de Astrofísica, Universidad de La Laguna, E-38206 La Laguna, Tenerife, Spain \\ ${ }^{9}$ University of Texas at Austin, McDonald Observatory, Fort Davis, TX 79734, USA; shetrone@ astro.as.utexas.edu \\ ${ }^{10}$ National Optical Astronomy Observatories, Tucson, AZ 85719, USA; cunha@email.noao.edu, vsmith@email.noao.edu \\ ${ }^{11}$ Department of Physics \& Astronomy and JINA, Joint Institute for Nuclear Astrophysics, \\ Michigan State University, East Lansing, MI 48824, USA; beers@ pa.msu.edu \\ 12 Apache Point Observatory, P.O. Box 59, Sunspot, NM 88349-0059, USA; dmbiz@ apo.nmsu.edu, gebelke@ apo.nmsu.edu, \\ viktorm@apo.nmsu.edu, elenam@apo.nmsu.edu, doravetz@apo.nmsu.edu, kpan@apo.nmsu.edu, asimmons@apo.nmsu.edu \\ ${ }^{13}$ Steward Observatory, University of Arizona, Tucson, AZ 85721, USA \\ ${ }^{14}$ Observatório Nacional, São Cristóvão, Rio de Janeiro, Brazil \\ ${ }^{15}$ Harvard-Smithsonian Center for Astrophysics, 60 Garden St., MS 20, Cambridge, MA 02138, USA; deisenstein@ cfa.harvard.edu \\ ${ }^{16}$ Department of Astronomy, The Ohio State University, Columbus, OH 43210, USA; jaj@astronomy.ohio-state.edu, gail.zasowski@gmail.com \\ ${ }^{17}$ Dunlap Institute for Astronomy and Astrophysics, University of Toronto, 50 St. George St., Toronto, Ontario M5S 3H4, Canada; drlaw@di.utoronto.ca \\ ${ }^{18}$ Gemini Observatory, 670 N. A’Ohoku Place, Hilo, HI 96720, USA; rpschiavon@ gmail.com \\ ${ }^{19}$ Astrophysics Research Institute, Liverpool John Moores University, Twelve Quays House, Egerton Wharf, Birkenhead CH41 ILD, UK \\ ${ }^{20}$ Department of Astronomy and Astrophysics, The Pennsylvania State University, University Park, PA 16802, USA; dps7@ psu.edu \\ ${ }^{21}$ Institute for Gravitation and the Cosmos, The Pennsylvania State University, University Park, PA 16802, USA \\ ${ }^{22}$ Department of Physics \& Astronomy, Johns Hopkins University, 3400 N. Charles St., Baltimore, MD 21218, USA; gail.zaswoski@ gmail.com \\ Received 2013 June 25; accepted 2013 September 16; published 2013 October 18
}

\begin{abstract}
The dynamics of the core of the Sagittarius (Sgr) dwarf spheroidal (dSph) galaxy are explored using high-resolution ( $R \sim 22,500$ ), $H$-band, near-infrared spectra of over 1000 giant stars in the central $3 \mathrm{deg}^{2}$ of the system, of which 328 are identified as Sgr members. These data, among some of the earliest observations from the Sloan Digital Sky Survey III/Apache Point Observatory Galactic Evolution Experiment (APOGEE) and the largest published sample of high resolution Sgr dSph spectra to date, reveal a distinct gradient in the velocity dispersion of Sgr from 11 to $14 \mathrm{~km} \mathrm{~s}^{-1}$ for radii $>0.8$ from center to a dynamical cold point of $8 \mathrm{~km} \mathrm{~s}^{-1}$ in the Sgr center-a trend differing from that found in previous kinematical analyses of Sgr over larger scales that suggests a more or less flat dispersion profile at these radii. Well-fitting mass models with either cored and cusped dark matter distributions can be found to match the kinematical results, although the cored profile succeeds with significantly more isotropic stellar orbits than required for a cusped profile. It is unlikely that the cold point reflects an unusual mass distribution. The dispersion gradient may arise from variations in the mixture of populations with distinct kinematics within the dSph; this explanation is suggested (e.g., by detection of a metallicity gradient across similar radii), but not confirmed, by the present data. Despite these remaining uncertainties about their interpretation, these early test data (including some from instrument commissioning) demonstrate APOGEE's usefulness for precision dynamical studies, even for fields observed at extreme airmasses.
\end{abstract}

Key words: galaxies: dwarf - galaxies: individual (Sagittarius dSph) - galaxies: interactions - galaxies: kinematics and dynamics - galaxies: stellar content - galaxies: structure

Online-only material: color figures

\section{INTRODUCTION}

The Sagittarius (Sgr) dwarf spheroidal (dSph) galaxy is a compelling Milky Way (MW) satellite for intense study, given (1) its unusual star formation and chemical enrichment history (e.g., Smecker-Hane \& McWilliam 2002; Siegel et al.
2007; Chou et al. 2010) and other properties (e.g., its own globular cluster system) evoking similarities to the Magellanic Clouds (e.g., Chou et al. 2010; Łokas et al. 2010b), but also (2) some remarkably unique physical properties. For example, Sgr presents the most vivid example of the MW's hierarchical growth via satellite accretion. It is also the only 
clear example of a nucleated dwarf galaxy among MW satellites, with a prominent metal-poor globular cluster (M54) possibly coinciding in phase space with its nucleus (e.g., Layden \& Sarajedini 2000; Majewski et al. 2003; Monaco et al. 2005b; Bellazzini et al. 2008, hereafter B08; but cf. Siegel et al. 2011). ${ }^{23}$

The latter fact is particularly germane to the debate over the dark matter distribution in dSph systems: While prevailing cold dark matter (CDM) models predict that their total density profiles should be cusped, their luminous density profiles are cored. Furthermore, several dynamical assessments of dSphs favor cored mass profiles.

1. Kleyna et al. (2003) argue that the double-peaked stellar structure of the Ursa Minor dSph (e.g., Palma et al. 2003) could only have survived a Hubble time if it lived within a host possessing a cored mass profile, whereas the dynamically cold, secondary clump would have been erased in less than a Gyr within a cusped mass distribution.

2. Calculations suggest that if the Fornax dSph had a cuspy mass profile its five globular clusters would have sunk to the center by dynamical friction in much less than a Hubble time (Goerdt et al. 2006; Sánchez-Salcedo et al. 2006).

3. Analysis of combined surface brightness profiles and velocity dispersions for some $\mathrm{dSph}$ galaxies lead to a preference for lower density, cored mass distributions (e.g., Gilmore et al. 2007; Battaglia et al. 2008).

If these implied cored profiles and inferred low central phase space densities are primordial and not the result of modification during the dynamical life of the satellite, a warm dark matter particle is implied (e.g., gravitinos, light sterile neutrinos), rather than one of the weakly interacting massive particle candidates of CDM (e.g., axions, neutralinos). On the other hand, B08 use $\mathrm{N}$-body simulations to argue that the presence of M54 in the very center of Sgr is compelling evidence for a cusped profile, which would have spiraled M54 to the nucleus by dynamical friction within a Hubble time if M54 had been born anywhere within $\sim 5 \mathrm{kpc}$ of center (see also Monaco et al. 2005b). However, isochrone fitting to precision photometry of M54 and the Sgr core from Advanced Camera for Surveys on the Hubble Space Telescope yields a $2 \mathrm{kpc}$ distance difference, implying that M54 may not be at the Sgr core, but merely projected upon it (Siegel et al. 2011). Clearly further work is needed to elucidate the true density profile shape for Sgr (and, by analogy, other $\mathrm{dSphs}$ ). Extensive, high accuracy velocity mapping of the $\mathrm{dSph}$ is expected to provide further data needed to help discriminate between competing mass models.

Nevertheless, despite Sgr's proximity, this dSph has seen surprisingly scant attention in terms of high-resolution spectroscopic study compared to other, more distant MW satellites. Because the system is of large angular size, even with the $\sim 1 \mathrm{deg}^{2}$ fields of view (FOVs) typical of many multifiber spectrographs, only pencil-beam samplings are possible, and these are typically at lower resolution. The most comprehensive Sgr spectroscopic studies at any resolution are those of Ibata et al. (1997; $R \sim 5000)$, B08 $(R \sim 5500)$, Peñarrubia et al. (2011; $R \sim 10,000)$, and Frinchaboy et al. (2012; $R \sim 15,000$ - hereafter F12), which together probe 24 independent directions and $\sim 3700$ different stars. The largest survey, F12, covers only $\sim 10 \%$ of the area within the isopleth corresponding to Sgr's $1800^{\prime}$ King limiting radius (Majewski et al.

\footnotetext{
23 That $\omega$ Centauri represents the remains of a similar, nucleated dwarf galaxy with a superposed globular cluster is an intriguing, but as yet not fully proven, hypothesis (B08; Carretta et al. 2010).
}

2003). Meanwhile, only a few dozen total stars have been explored at "echelle" resolution across the dwarf by the combined studies of Smecker-Hane \& McWilliam (2002), Monaco et al. (2005a), Chou et al. (2010), and Sbordone et al. (2007). ${ }^{24}$ As a result, we know comparably little about the detailed abundance distributions and dynamics of the core of this intriguing system compared to those of other classical MW dSphs where velocity dispersion profiles and chemical abundance patterns are derived with hundreds to thousands of stellar members (e.g., Walker et al. 2007).

This situation will surely soon change if any of the several available multiobject, high-resolution spectrographs are devoted to large-scale surveys of this most interesting Galactic satellite. In the meantime, a significant high-resolution spectroscopic sample of spectra of Sgr has already been obtained by the Sloan Digital Sky Survey III (SDSS-III; Eisenstein et al. 2011) as a byproduct of commissioning and early survey observations by the Apache Point Observatory Galactic Evolution Experiment (APOGEE). These high-resolution, $H$-band spectra lie at interesting positions probing Sgr between the intense central nucleus study of B08 and the larger radii probed by most other Sgr surveys. We use these APOGEE spectra to show, for the first time, that within $1^{\circ}$ of its center the Sgr dSph contains a strong velocity dispersion gradient and a modest $[\mathrm{Fe} / \mathrm{H}]$ gradient.

\section{APOGEE SPECTRA OF SGR AND THEIR REDUCTION}

The APOGEE project is described in Allende Prieto et al. (2008), Majewski et al. (2010), and Eisenstein et al. (2011). It uses a cryogenic, bench-mounted spectrograph recording 300 simultaneous spectra covering $\lambda \lambda 1.51-1.70 \mu \mathrm{m}$ for light fed to it from the Sloan $2.5 \mathrm{~m}$ telescope (Gunn et al. 2006) via $40 \mathrm{~m}$ long optical fibers. The spectrograph, described by Wilson et al. (2010), records three distinct spectral regions onto separate detectors (spanning 1.51-1.58, 1.59-1.64, and 1.65-1.70 $\mu \mathrm{m}$, respectively) at $R \sim 22,500$.

The $2^{\prime \prime}$ diameter APOGEE fibers are plugged into standard Sloan plugplates and observed similarly to the optical Sloan surveys (e.g., Smee et al. 2013), but with these variations (see Zasowski et al. 2013): (1) 35 fibers in each plugplate configuration are used to collect sky/airglow spectra, (2) another 35 fibers target bright $(5<H<11)$, hot (generally $\left[J-K_{s}\right]<$ 0.4) stars to monitor telluric $\left(\mathrm{H}_{2} \mathrm{O}, \mathrm{CO}_{2}, \mathrm{CH}_{4}\right)$ absorption, and (3) because the extreme zenith distances at Apache Point Observatory (APO) required to observe the Sgr core $\left(63^{\circ}\right)$ impose strong differential refraction effects, Sgr plugplates were tested with various field sizes and magnitude limits. Table 1 (see also Figure 1) summarizes the Sgr core plugplates observed and when, their field centers and diameters (FOV), $H$-band limit $\left(H_{\text {lim }}\right)$ for stars targeted with standard APOGEE criteria (see below) and for the faintest Sgr member, total integration time, number of Sgr members ending up in our final dynamical analysis (see below), total number of stars with measured radial velocities (RVs), and their median RV error. Observations of plate 5100 were taken before the instrument was in its fully commissioned state and with elements in nonoptimal alignment, which led to slightly blurred line-spread functions degrading the resolution of the $1.65-1.70 \mu \mathrm{m}$ region to only $R \sim 14,500$; therefore, to use the highest quality spectral regions and maintain consistency, analysis of all spectra

\footnotetext{
24 While B08's Sgr+M54 survey did include some stars observed at $R \sim 17,000$ with FLAMES, these were primarily M54 stars and their entire survey was concentrated within $9^{\prime}$ of center.
} 

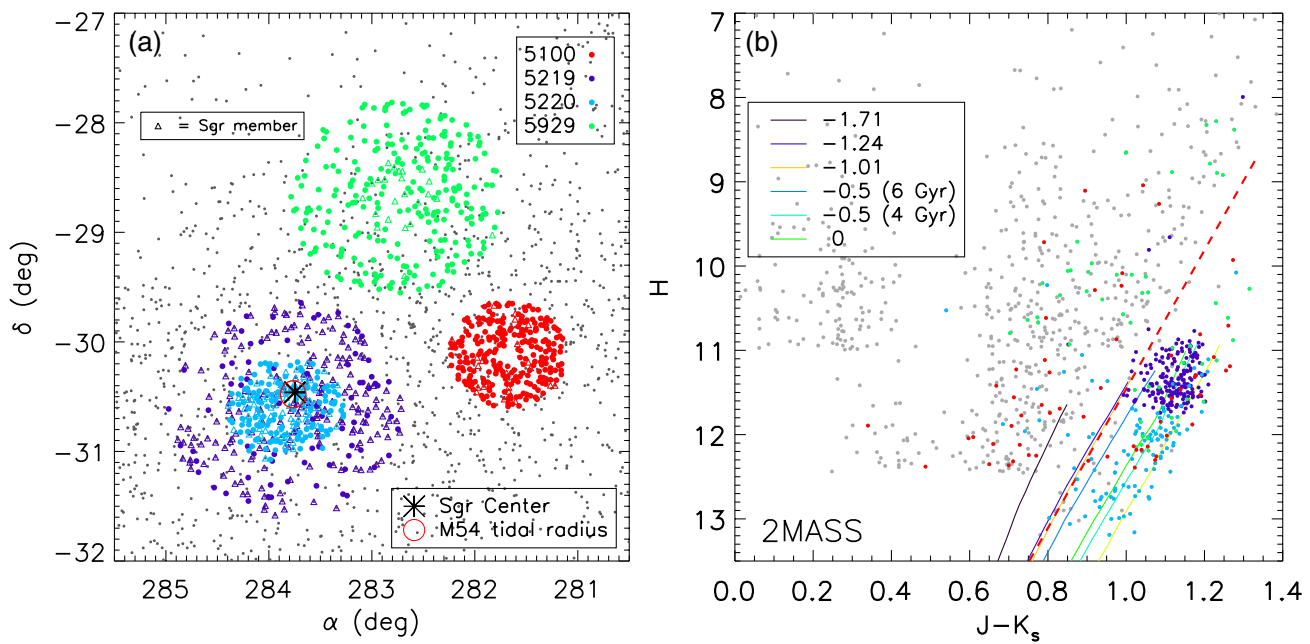

Figure 1. Sky (left) and 2MASS color-magnitude (right) distributions of observed stars in Sgr, color-coded by plugplate number (but only for stars with $90<v_{\mathrm{GSR}}<220 \mathrm{~km} \mathrm{~s}^{-1}$ in the right panel). The left panel also shows the Majewski et al. (2003) distribution of M giant stars (gray points) and fitted center (black star) for Sgr as well as the 7'.4 tidal radius of M54 (B08). The CMD includes Padova isochrones (Bressan et al. 2012) corresponding to major Sgr populations identified by Siegel et al. (2007) and our limit for accepting potential Sgr members (red dashed line).

(A color version of this figure is available in the online journal.)

Table 1

APOGEE Observations in the Field of the Sagittarius dSph Galaxy

\begin{tabular}{|c|c|c|c|c|c|c|c|c|}
\hline Plate & UT Date & $\begin{array}{l}(l, b) \\
(\operatorname{deg})\end{array}$ & FOV & $H_{\text {lim }}($ plate $/ \mathrm{Sgr})$ & $\begin{array}{l}\text { Int } \\
\text { (s) }\end{array}$ & Members & RVs & $\begin{array}{c}\epsilon_{\mathrm{RV}} \\
\left(\mathrm{km} \mathrm{s}^{-1}\right)\end{array}$ \\
\hline 5100 & $2011 \mathrm{Jul} 18$ & $(5.2,-12.3)$ & $1^{\circ}$ & $12.5 / 12.4$ & 4729 & 24 & 262 & 0.14 \\
\hline 5219 & 2011 Sep 6,7 & $(5.5,-14.2)$ & $2^{\circ}$ & $11.0 / 11.7$ & 8004 & 190 & 265 & 0.15 \\
\hline 5220 & 2012 Jun 5,12 & $(5.5,-14.2)$ & $1^{\circ}$ & $12.2 / 13.2$ & 8004 & 91 & 218 & 0.30 \\
\hline 5929 & 2012 Jun 6 & $(6.9,-12.6)$ & $2^{\circ}$ & $11.0 / 12.2$ & 4002 & 23 & 262 & 0.09 \\
\hline
\end{tabular}

was confined to $1.51-1.64 \mu \mathrm{m}$, a region where, in any case, relevant information density happens to concentrate. Targets in plates 5219, 5220, and 5929 were selected from Sgr members identified by F12, with leftover fibers filled by random sampling $(J-K)_{0} \geqslant 0.5$ stars from the Two Micron All Sky Survey (2MASS) point source catalog ${ }^{25}$ as described in Zasowski et al. (2013); targets in plate 5100 were selected using only the latter method (Figure 1).

An automated data reduction pipeline written specifically for APOGEE (D. L. Nidever et al., in preparation) was used to convert the raw APOGEE data cubes into one-dimensional, wavelength-calibrated spectra and derive RVs previously shown to be accurate to $0.26 \pm 0.22 \mathrm{~km} \mathrm{~s}^{-1}$ (see Nidever et al. 2012). For 46 stars in common between plugplates 5219 and 5220 the dispersion in difference between derived RVs is $0.29 \mathrm{~km} \mathrm{~s}^{-1}$.

Metallicities $([\mathrm{Fe} / \mathrm{H}])$ are derived using an automated method with the APOGEE Stellar Parameters and Chemical Abundances Pipeline (ASPCAP; A. E. García Pérez et al., in preparation). The version of ASPCAP code used for the present analysis derives stellar atmospheric parameters via $\chi^{2}$ fits of the airglow-masked APOGEE spectra against spectra interpolated in libraries of synthetic spectra at the observed resolution. For the $\mathrm{K}$ and $\mathrm{M}$ spectral classes of interest here the code uses a six-dimensional library with dimensions spanning ranges: (1) $3500 \leqslant T_{\text {eff }} \leqslant 5000 \mathrm{~K},(2) 0.0 \leqslant \log g \leqslant 5.0,(3)-2.5 \leqslant$ $[\mathrm{Fe} / \mathrm{H}] \leqslant+0.5,(4)-1.0 \leqslant[\mathrm{C} / \mathrm{Fe}] \leqslant+1.0,(5)-1.0 \leqslant$ $[\mathrm{N} / \mathrm{Fe}] \leqslant+1.0$, and $(6)-1.0 \leqslant[\alpha / \mathrm{Fe}] \leqslant+1.0$. The library

25 The 2MASS photometry was dereddened using the RJCE method (Majewski et al. 2011) with WISE (Wright et al. 2010) providing the required mid-infrared photometry. is based on ATLAS9 model atmospheres (Castelli \& Kurucz 2004) and spectral synthesis calculations with the code ASS $\epsilon$ T (Koesterke et al. 2008) and an atomic line list optimized to match the solar spectrum. The microturbulence was fixed at $\xi=2 \mathrm{~km} \mathrm{~s}^{-1}$. Tests of the $[\mathrm{Fe} / \mathrm{H}]$ delivered by this version of ASPCAP code were made via APOGEE observations of 20 open and globular clusters having high-quality metallicities in the literature. From this assessment we find that the ASPCAP $[\mathrm{Fe} / \mathrm{H}]$ are reliable to $0.06-0.10 \mathrm{dex}$ when compared to literature values at solar metallicity, and to $0.10-0.15 \mathrm{dex}$ at $[\mathrm{Fe} / \mathrm{H}] \leqslant-1$.

\section{DYNAMICS OF SAGITTARIUS CORE STARS}

Figures 2(a) and (b) show the distribution of Galactic Standard of Rest (GSR) RVs $\left(v_{\mathrm{GSR}}\right)$ for the APOGEE observations of 1007 stars in the Sgr fields, assuming a solar motion in righthanded Galactic coordinates of $(+10.0,+225.3,+7.2) \mathrm{km} \mathrm{s}^{-1}$. The different relative strengths of the narrow "Sgr peak" in the inner versus outer two fields (Figure 2(b)) reflects the relative densities of Sgr to MW field stars at the two radii and the fact that known Sgr stars were deliberately targeted only in the center-field observations.

To determine metallicity and velocity dispersion profiles for Sgr, we winnow the data to a more pure sample of Sgr members by first applying a broad kinematical selection of stars in the "Sgr peak" $\left(90<v_{\mathrm{GSR}}<220 \mathrm{~km} \mathrm{~s}^{-1}\right.$; colored points in the Figure 1 color-magnitude diagram, CMD) followed by a color-magnitude limit (Figure 1) roughly tracking an $[\mathrm{Fe} / \mathrm{H}]=-1$ isochrone. To this sample we then apply a $3 \sigma_{v}$ iterative outlier rejection scheme to the velocities. The color 


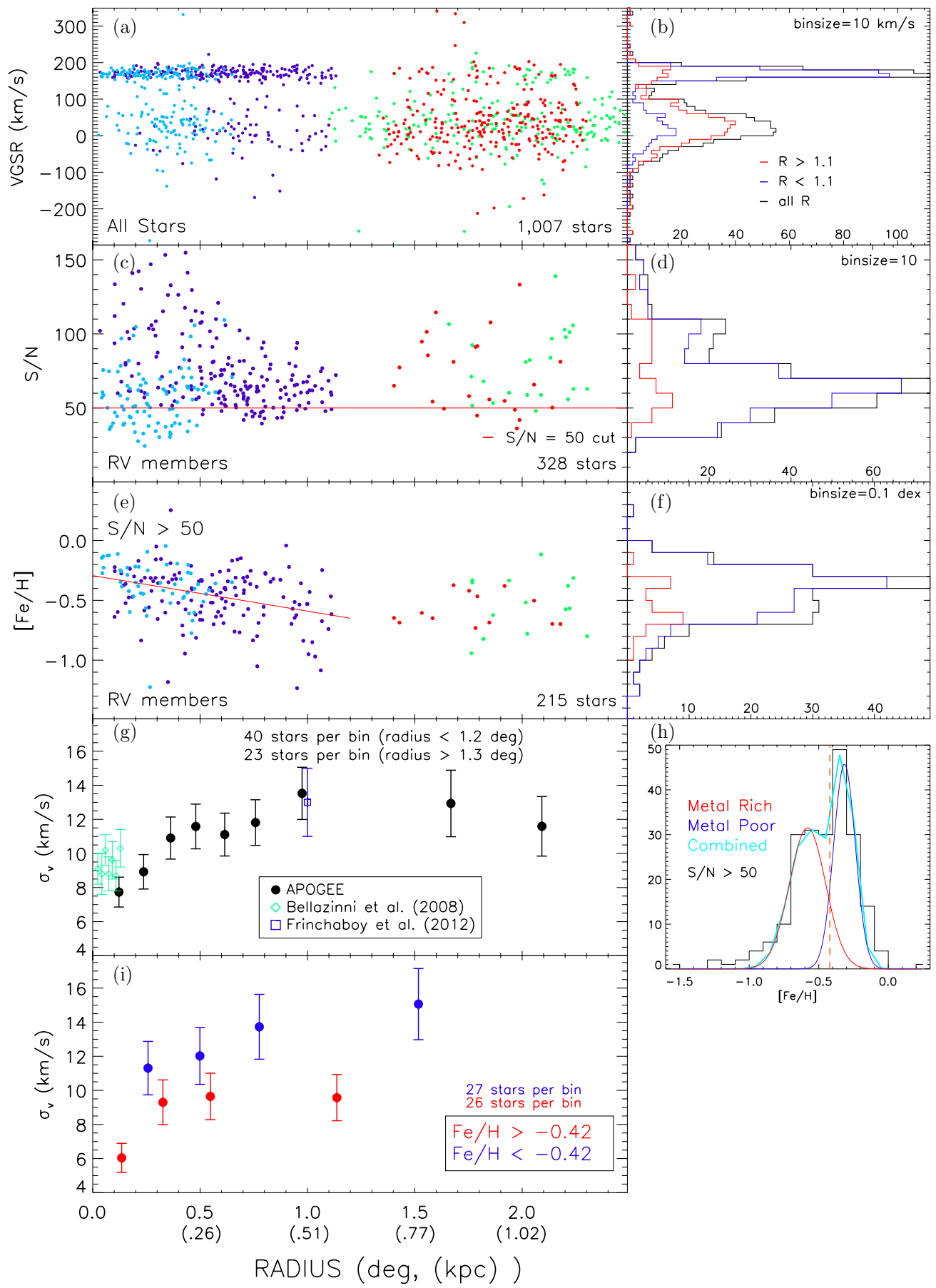

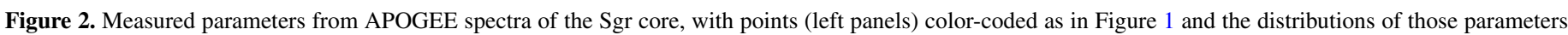

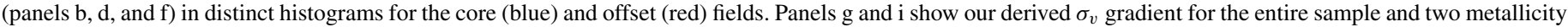
subsamples (defined by the Gaussian fits in panel h), respectively, compared to results from F12 and B08 (panel g).

(A color version of this figure is available in the online journal.)

and $3 \sigma_{v}$ constraints actually remove a relatively small fraction of stars in the "Sgr velocity peak," but help reduce contamination from M54 (defined by B08 to span $-1.8<[\mathrm{Fe} / \mathrm{H}]<-1.1$, and eliminating one star within 7.'4 of M54 center) and MW field stars, at the risk of a slight, $[\mathrm{Fe} / \mathrm{H}] \gtrsim-1$ bias (see isochrones in Figure 1). All 328 stars remaining have sufficient signalto-noise ratio (S/N; Figures 2(c) and (d)) to have extremely reliable RVs (precision $\ll 1 \mathrm{~km} \mathrm{~s}^{-1}$ ) for measuring velocity dispersions $\left(\sigma_{v}\right)$.

The resulting $\operatorname{Sgr} \sigma_{v}$ profile (Figure 2(g)) shows dramatically, and for the first time, that the heart of Sgr is characterized by a steady and definitive gradient from $11-14 \mathrm{~km} \mathrm{~s}^{-1}$ for stars at $>0.8$ radius to a dynamically colder center at $<8 \mathrm{~km} \mathrm{~s}^{-1}$ (and with no perceptible rotation). As summarized by F12, Sgr velocity dispersions measured by other surveys are generally higher than most values shown in Figure 2(g), particularly for at least the next several degrees in radius. However, only the studies of Ibata et al. (1997) and F12 have sufficient statistics to derive velocity dispersions in multiple Sgr fields, with the F12 data of much better quality due to higher spectral resolution ( $R=15,000$ versus 5000 in Ibata et al.). For comparison, we include in Figure 2(g) the $\sigma_{v}$ measures of those nearby fields 

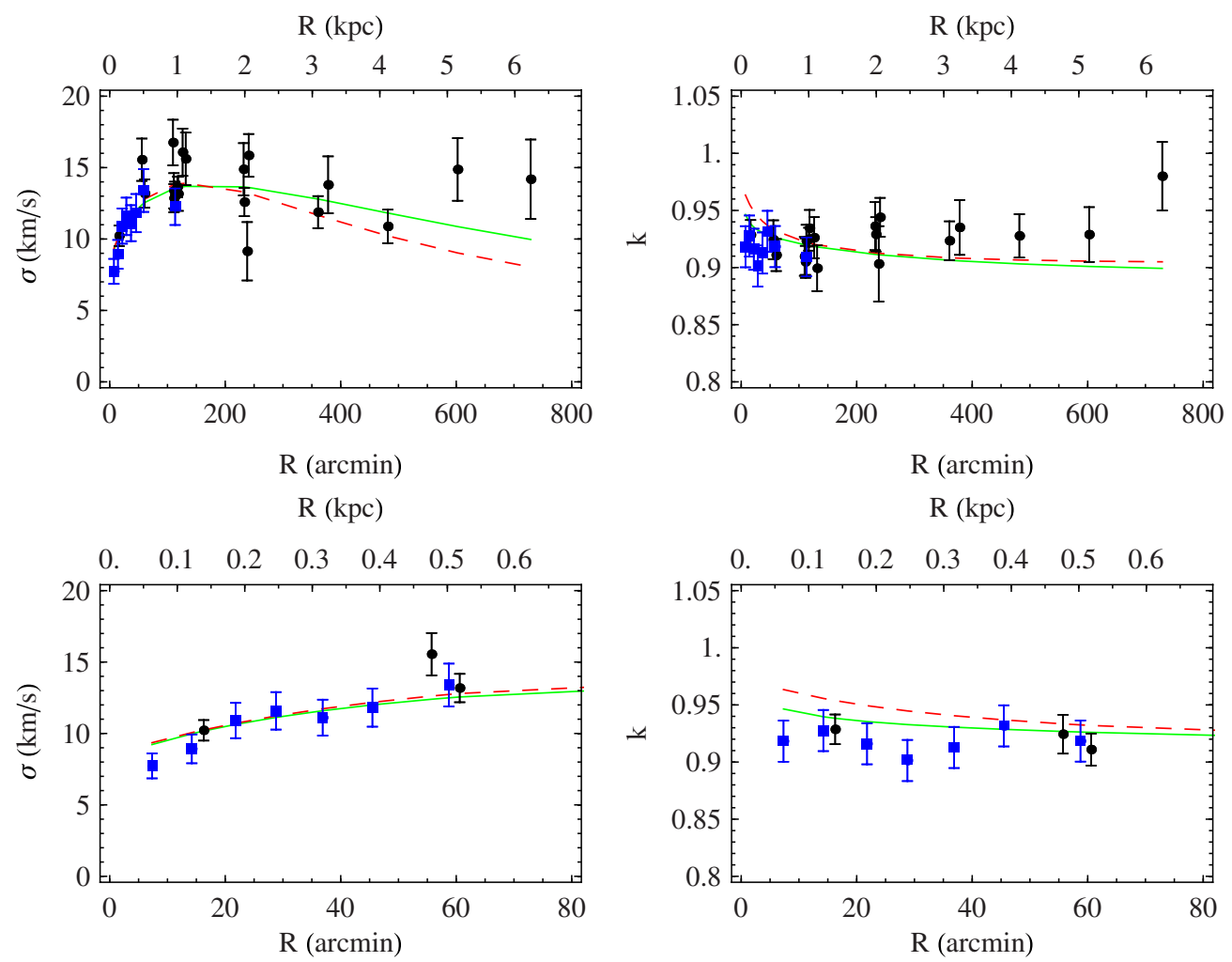

Figure 3. Velocity dispersion (left panels) and kurtosis (right panels) data for the Sgr core from F12 (black circles; we exclude MW-contaminated fields discussed by F12), and from APOGEE data (blue squares). The green solid (red dashed) line is the best fit of a two-component (stars and dark matter) model with cuspy (cored) dark matter distribution and constant anisotropy parameter, $\beta$. The best-fitting cuspy (cored) model has a total mass of $8.1(5.6) \times 10^{8} M_{\odot}$ and $\beta=-0.7(-0.3)$.

(A color version of this figure is available in the online journal.)

with the best data from F12 and B08 (who analyzed 1152 Very Large Telescope/FLAMES and Keck/DEMOS spectra of M54 and the inner $9^{\prime}$ of Sgr). The APOGEE observations seemingly "connect" the warmer F12 and colder B08 points, though we find a somewhat smaller central Sgr velocity dispersion than B08, which may relate to APOGEE's order of magnitude smaller intrinsic velocity uncertainties. The observed gradient should not be the result of M54 contamination, given our elimination of $[\mathrm{Fe} / \mathrm{H}] \lesssim-1$ stars by the CMD selection and M54's tidal radius of $\sim 7$ '.4 (B08; Figure 1 (a)).

\section{EXPLAINING THE DYNAMICAL COLD POINT}

That Sgr has a strong increase in velocity dispersion with radius makes it similar to other MW dSphs with analogous $\sigma_{v}$ trends, such as Draco, Sextans, and Carina (Walker et al. 2007), although the causes for such features likely vary. For example, Muñoz et al. (2008) have suggested that in Carina the phenomenon is related to tidal disruption, and while Sgr is also obviously tidally disrupting, the radii that would be affected by this should be much farther out than the region probed here (the Figure 2(g) gradient lies within Sgr's innermost $0.5 \mathrm{kpc}$, well inside its several kiloparsec tidal radius; Law \& Majewski 2010).

However, the observed gradient in the velocity dispersion profile of Sgr might naturally be explained in terms of its underlying mass distribution. Figure 3 shows the profiles of velocity dispersion and a kurtosis-like variable $\left(k=[\log (3 \kappa / f)]^{1 / 10}\right.$, where $\kappa$ is the standard estimator of the RV distribution kurtosis, and the correction $f$ depends on the number of stars per bin - see Appendix of Łokas \& Mamon 2003) calculated from a combination of our data and the best data from F12. The higher order moment of velocity dispersion represented by $k$ offers an additional sensitive constraint on the mass models. Together, these data were fit by Jeans equations solutions (see Łokas et al. 2005) assuming a two-component model with the stellar distribution approximated by a Sérsic profile (with parameters from Łokas et al. 2010b) and the dark matter distribution by $\rho=C r^{-\alpha} \exp \left(-r / r_{b}\right)$. We considered two inner slopes, $\alpha=0$ (core) and $\alpha=1$ (cusp), and adjusted the total dark mass, cutoff scale $r_{b}$ and stellar orbit anisotropy parameter, $\beta$, assumed to be constant with radius.

The cuspy dark matter profile (green lines in Figure 3) fits the data slightly better than the profile with the core (red lines in Figure 3) with $M / L$ within $R \lesssim 600$ arcmin of 40.2 versus 33.8 and reduced $\chi^{2}$ of 1.3 versus 1.7 (although neither model seems to match well for $R \gtrsim 600^{\prime}$ ). In spite of resorting to modeling the higher velocity moments, the degeneracy between the parameters of the dark matter profile (inner slope and characteristic scale) remains (see Łokas \& Mamon 2003; Agnello \& Evans 2012). However, the best fit of the cored profile is achieved with more isotropic orbits $(\beta=-0.3)$ than for the cuspy one $(\beta=-0.7)$. Interestingly, isotropic, or even mildly radial $(\beta \gtrsim 0)$ stellar orbits are predicted in the context of the tidal stirring model for the formation of $\mathrm{dSph}$ galaxies in the Local Group. As shown in Łokas et al. (2010a) and Kazantzidis et al. (2011), strongly tidally affected dwarfs are dominated by mildly radial orbits as a result of bar formation at the first pericenter passage on their orbit around the MW. This should also be the case for Sgr if it formed from a disky progenitor, as proposed by Łokas et al. (2010b).

Another explanation suggested (e.g., McConnachie et al. 2007) to account for systematically smaller velocity dispersions in $\mathrm{dSph}$ cores is the presence of variations in the mixing of 
distinct stellar populations with differing kinematics. MW dSph galaxies are commonly found to have multiple populations (e.g., Majewski et al. 1999; Harbeck et al. 2001; Tolstoy et al. 2004; Ibata et al. 2006), and Sgr is no exception (e.g., Siegel et al. 2007). Moreover, from the APOGEE data, we verify that, at minimum, there is a small but identifiable metallicity gradient within the inner few degrees of Sgr (Figure 2(e)), evident as a few tenths of a dex drop in $[\mathrm{Fe} / \mathrm{H}]$ from the center to offset APOGEE fields (Figure 2(f)). To assess this $[\mathrm{Fe} / \mathrm{H}]$ distribution we have used only those 215 stars with the most reliable ASPCAP data: those with $T_{\text {eff }}>3575 \mathrm{~K}$ and having spectra with total $\mathrm{S} / \mathrm{N}>50$ pixel $^{-1}$. The existence of an $[\mathrm{Fe} / \mathrm{H}]$ gradient is consistent with previous analyses of the Sgr system (e.g., Alard 2001; Chou et al. 2010) that show an overall general metallicity increase toward the Sgr center.

Although slightly shaped by the combined effects of the color-magnitude selection and the ASPCAP temperature limit discussed above, the bulk of the stars in this pruned APOGEE sample have metallicities distributed across the $-0.8<$ $[\mathrm{Fe} / \mathrm{H}]<-0.2$ range identified with intermediate age and metallicity stars ("SInt") and exhibiting several subpopulations in the population synthesis of Siegel et al. (2007). The APOGEE metallicity distribution (Figure 2(h)) also hints at possible subgroups, and a two-Gaussian fit suggests $[\mathrm{Fe} / \mathrm{H}] \sim-0.42$ as a reasonable, though approximate, division between them. These sample subdivisions show some differences in $\sigma_{v}$ profile, with the metal-poor subsample exhibiting consistently larger $\sigma_{v}$ within the central few degrees of Sgr compared to the metal-rich subsample. That each metallicity subsample still internally shows a dispersion gradient may belie the fact that our simple basis for separating population subsamples is not the cleanest. On the other hand, the Figure 3 fits find velocity distributions in each radial bin consistent (within the errors) with Gaussian (defined as $k=0.93$ ), whereas McConnachie et al. (2007) suggest that dwarf galaxies having mixed stellar populations in equilibrium should yield leptokurtic velocity distributions at radii where multiple populations make a significant contribution.

Derivations of the abundances of additional chemical elements expressed within the APOGEE spectra will hopefully improve our ability to sort Sgr core stars by population and test whether the observed dynamics involves the interplay of populations with distinct orbital characteristics. Although such a populations-based approach may presently be insufficient to explain the velocity dispersion gradient in a way envisioned by McConnachie et al., ultimately unraveling this interplay may be the best path to a definitive assessment of the underlying mass distribution. As pointed out by Walker \& Peñarrubia (2011; see also Agnello \& Evans 2012), that each subpopulation is in equilibrium within the same gravitational potential can be used to measure directly the slope of the density profile using simple mass estimators-but only if the triaxial orientation of the satellite can be determined (Kowalczyk et al. 2013). The latter is a prospect, however, that is particularly promising for the Sgr system (Łokas et al. 2010b; Kowalczyk et al. 2013).

We gratefully acknowledge support from National Science Foundation (NSF) grant AST11-09718 and the Polish National Science Centre under grant NN203580940 to E.L.E.

Funding for SDSS-III has been provided by the Alfred P. Sloan Foundation, the Participating Institutions, the National Science Foundation, and the US Department of Energy Office of Science. The SDSS-III Web site is http://www.sdss3.org/.
SDSS-III is managed by the Astrophysical Research Consortium for the Participating Institutions of the SDSS-III Collaboration including the University of Arizona, the Brazilian Participation Group, Brookhaven National Laboratory, University of Cambridge, Carnegie Mellon University, University of Florida, the French Participation Group, the German Participation Group, Harvard University, the Instituto de Astrofisica de Canarias, the Michigan State/Notre Dame/JINA Participation Group, Johns Hopkins University, Lawrence Berkeley National Laboratory, Max Planck Institute for Astrophysics, Max Planck Institute for Extraterrestrial Physics, New Mexico State University, New York University, Ohio State University, Pennsylvania State University, University of Portsmouth, Princeton University, the Spanish Participation Group, University of Tokyo, University of Utah, Vanderbilt University, University of Virginia, University of Washington, and Yale University.

\section{REFERENCES}

Agnello, A., \& Evans, N. W. 2012, ApJL, 754, L39

Alard, C. 2001, A\&A, 377, 389

Allende Prieto, C., Majewski, S. R., Schiavon, R., et al. 2008, AN, 329, 1018

Battaglia, G., Helmi, A., Tolstoy, E., et al. 2008, ApJL, 681, L13

Bellazzini, M., Ibata, R. A., Chapman, S. C., et al. 2008, AJ, 136, 1147 (B08)

Bressan, A., Marigo, P., Girardi, L., et al. 2012, MNRAS, 427, 127

Carretta, E., Bragaglia, A., Gratton, R. G., et al. 2010, ApJL, 714, L7

Castelli, F., \& Kurucz, R. L. 2004, in IAU Symp. 210, Modelling of Stellar Atmospheres, ed. N. Piskunov, W. W. Weiss, \& D. F. Gray (San Francisco, CA: ASP), poster A20

Chou, M.-Y., Cunha, K., Majewski, S. R., et al. 2010, ApJ, 708, 1290

Eisenstein, D. J., Weinberg, D. H., Agol, E., et al. 2011, AJ, 142, 72

Frinchaboy, P. M., Majewski, S. R., Muñoz, R. R., et al. 2012, ApJ, 756, 74 (F12)

Gilmore, G., Wilkinson, M. I., Wyse, R. F. G., et al. 2007, ApJ, 663, 948

Goerdt, T., Moore, B., Read, J. I., Stadel, J., \& Zemp, M. 2006, MNRAS, 368, 1073

Gunn, J. E., Siegmund, W. A., Mannery, E. J., et al. 2006, AJ, 131, 2332

Harbeck, D., Grebel, E. K., Holtzman, J., et al. 2001, AJ, 122, 3092

Ibata, R., Chapman, S., Irwin, M., Lewis, G., \& Martin, N. 2006, MNRAS, 373, L70

Ibata, R. A., Wyse, R. F. G., Gilmore, G., Irwin, M. J., \& Suntzeff, N. B. 1997, AJ, 113, 634

Kazantzidis, S., Łokas, E. L., Callegari, S., Mayer, L., \& Moustakas, L. A. 2011, ApJ, 726, 98

Kleyna, J. T., Wilkinson, M. I., Gilmore, G., \& Evans, N. W. 2003, ApJL, $588, \mathrm{~L} 21$

Koesterke, L., Allende Prieto, C., \& Lambert, D. L. 2008, ApJ, 680, 764

Kowalczyk, K., Lokas, E. L., Kazantzidis, S., \& Mayer, L. 2013, MNRAS, 431, 2796

Law, D. R., \& Majewski, S. R. 2010, ApJ, 714, 229

Layden, A. C., \& Sarajedini, A. 2000, AJ, 119, 1760

Łokas, E. L., Kazantzidis, S., Klimentowski, J., Mayer, L., \& Callegari, S. 2010a, ApJ, 708, 1032

Łokas, E. L., Kazantzidis, S., Majewski, S. R., et al. 2010b, ApJ, 725, 1516

Łokas, E. L., \& Mamon, G. A. 2003, MNRAS, 343, 401

Łokas, E. L., Mamon, G. A., \& Prada, F. 2005, MNRAS, 363, 918

Majewski, S. R., Siegel, M. H., Patterson, R. J., \& Rood, R. T. 1999, ApJL, 520, L33

Majewski, S. R., Skrutskie, M. F., Weinberg, M. D., \& Ostheimer, J. C. 2003, ApJ, 599, 1082

Majewski, S. R., Wilson, J. C., Hearty, F., Schiavon, R. R., \& Skrutskie, M. F. 2010, in IAU Symp. 265, Chemical Abundances in the Universe: Connecting First Stars to Planets, ed. K. Cunha, M. Spite, \& B. Barbuy (Cambridge: Cambridge Univ. Press), 480

Majewski, S. R., Zasowski, G., \& Nidever, D. L. 2011, ApJ, 739, 25

McConnachie, A. W., Peñarrubia, J., \& Navarro, J. F. 2007, MNRAS, 380, L75

Monaco, L., Bellazzini, M., Bonifacio, P., et al. 2005a, A\&A, 441, 141

Monaco, L., Bellazzini, M., Ferraro, F. R., \& Pancino, E. 2005b, MNRAS, 356,1396

Muñoz, R. R., Majewski, S. R., \& Johnston, K. V. 2008, ApJ, 679, 346

Nidever, D. L., Zasowski, G., Majewski, S. R., et al. 2012, ApJL, 755, L25 
Palma, C., Majewski, S. R., Siegel, M. H., et al. 2003, AJ, 125, 1352

Peñarrubia, J., Zucker, D. B., Irwin, M. J., et al. 2011, ApJL, 727, L2

Sánchez-Salcedo, F. J., Reyes-Iturbide, J., \& Hernandez, X. 2006, MNRAS, 370,1829

Sbordone, L., Bonifacio, P., Buonanno, R., et al. 2007, A\&A, 465, 815

Siegel, M. H., Dotter, A., Majewski, S. R., et al. 2007, ApJL, 667, L57

Siegel, M. H., Majewski, S. R., Law, D. R., et al. 2011, ApJ, 743, 20

Smecker-Hane, T. A., \& McWilliam, A. 2002, arXiv:astro-ph/0205411
Smee, S., Gunn, J. E., Uomoto, A., et al. 2013, AJ, 146, 32

Tolstoy, E., Irwin, M. J., Helmi, A., et al. 2004, ApJL, 617, L119

Walker, M. G., Mateo, M., Olszewski, E. W., et al. 2007, ApJS, 171, 389

Walker, M. G., \& Peñarrubia, J. 2011, ApJ, 742, 20

Wilson, J. C., Hearty, F., Skrutskie, M. F., et al. 2010, Proc. SPIE, 7735, 46

Wright, E. L., Eisenhardt, P. R. M., Mainzer, A. K., et al. 2010, AJ, 140, 1868

Zasowski, G., Johnson, J. A., Frinchaboy, P. M., et al. 2013, AJ, 146, 81 